Georgian Mathematical Journal

Volume 12 (2005), Number 2, 255-260

\title{
ON ABSOLUTELY NEGLIGIBLE SETS IN UNCOUNTABLE SOLVABLE GROUPS
}

\author{
ALEXANDER KHARAZISHVILI
}

\begin{abstract}
Absolutely negligible sets in uncountable groups are considered in connection with the measure extension problem (for $\sigma$-finite invariant or quasi-invariant measures). In particular, it is proved that, for any uncountable solvable group $(G, \cdot)$, there exists a countable covering of $G$ consisting of $G$-absolutely negligible sets.
\end{abstract}

2000 Mathematics Subject Classification: 28A05, 28D05.

Key words and phrases: Solvable group, invariant measure, quasi-invariant measure, absolutely negligible set, Sierpiński's problem.

Let $(G, \cdot)$ be an arbitrary group. In our further consideration, we will identify $G$ with the group of all left translations of $G$.

A measure $\mu$ on $G$ is called $G$-invariant if $\mu$ is defined on a $G$-invariant $\sigma$ algebra of subsets of $G$ and $\mu(g(X))=\mu(X)$ for any element $g \in G$ and any set $X \in \operatorname{dom}(\mu)$.

A measure $\mu$ on $G$ is called $G$-quasi-invariant if $\mu$ is defined on a $G$-invariant $\sigma$-algebra of subsets of $G$ and

$$
\mu(g(X))=0 \Leftrightarrow \mu(X)=0
$$

for any element $g \in G$ and any set $X \in \operatorname{dom}(\mu)$.

As is known, if $\mu$ is a nonzero $\sigma$-finite $G$-invariant ( $G$-quasi-invariant) measure on an uncountable group $(G, \cdot)$, then the domain of $\mu$ differs from the family of all subsets of $G$ (see, for instance, [1] and [2]). In this connection, the following natural problem arises: does there exist a $G$-invariant ( $G$-quasi-invariant) measure $\mu^{\prime}$ on $G$ strictly extending $\mu$ ? In the classical case where $G$ coincides with the $n$-dimensional Euclidean space $R^{n}(n \geq 1)$ and $\mu$ is an invariant extension of the standard Lebesgue measure on $R^{n}$, this problem was originally posed by Sierpiński (see, e.g., [3]).

There are various aspects of the above-mentioned Sierpiński problem and of its generalized version for an arbitrary uncountable group $(G, \cdot)$. In the present paper, we are going to discuss an important aspect of this problem, closely connected with the concept of an absolutely negligible set. We believe that the notion of an absolutely negligible set is of deep interest from the measuretheoretical and group-theoretical points of view and plays a significant role in studying questions concerning extensions of invariant (quasi-invariant) measures.

Let $X$ be a subset of $(G, \cdot)$. We say that $X$ is $G$-absolutely negligible (in $G$ ) if, for any $\sigma$-finite $G$-invariant (respectively, $G$-quasi-invariant) measure $\mu$ on 
$G$, there exists a $G$-invariant (respectively, $G$-quasi-invariant) measure $\mu^{\prime}$ on $G$ extending $\mu$ and satisfying the relation $\mu^{\prime}(X)=0$.

This definition implies at once that if $X$ is a $G$-absolutely negligible set in $G$ and $X$ does not belong to the domain of an initial measure $\mu$, then $\mu$ is strictly extendible by using this $X$. The said above immediately leads to the following method of extending $\mu$. Denote by $\omega$ the first infinite cardinal and suppose that a given group $G$ admits a countable covering $\left\{X_{n}: n<\omega\right\}$ such that all sets $X_{n}(n<\omega)$ are $G$-absolutely negligible in $G$. If our measure $\mu$ is not identically equal to zero, then there exists at least one $n<\omega$ for which the set $X_{n}$ does not belong to $\operatorname{dom}(\mu)$. Consequently, our $\mu$ can be strictly extended with the aid of $X_{n}$.

In this way, we come to the following problem, closely connected with the generalized version of Sierpiński's problem.

Problem. Give a characterization of all those uncountable groups $(G, \cdot)$ which admit a countable covering consisting of $G$-absolutely negligible sets.

This problem still remains open. The main goal of the present article is to demonstrate that any uncountable solvable group $(G, \cdot)$ can be covered by a countable family of $G$-absolutely negligible sets.

For this purpose, we need several auxiliary propositions.

Lemma 1. Let $(G, \cdot)$ be an arbitrary group and let $X$ be a subset of $G$. The following two assertions are equivalent:

1) $X$ is G-absolutely negligible;

2) for any countable family $\left\{g_{i}: i \in I\right\} \subset G$, there exists a countable family $\left\{f_{j}: j \in J\right\} \subset G$ such that

$$
\bigcap_{j \in J} f_{j}\left(\bigcup_{i \in I} g_{i} X\right)=\varnothing .
$$

Lemma 1 yields a purely algebraic characterization of $G$-absolutely negligible sets in $G$. In particular, it immediately follows from this lemma that if $X$ is a $G$-absolutely negligible subset of $G$, then

$$
\mu_{*}\left(\cup\left\{g_{i} X: i \in I\right\}\right)=0
$$

for every countable family $\left\{g_{i}: i \in I\right\}$ of elements of $G$ and for every $\sigma$-finite $G$-quasi-invariant measure $\mu$ on $G$.

A detailed proof of Lemma 1 can be found in [4] or [5] where a more general result is also presented concerning a similar characterization of absolutely negligible sets in an abstract space $E$ equipped with some group $G$ of transformations of $E$.

Lemma 2. Let $G$ and $H$ be two groups and let

$$
\phi: G \rightarrow H
$$

be a surjective homomorphism. Suppose that $Y$ is an $H$-absolutely negligible subset of $H$. Then the set $X=\phi^{-1}(Y)$ is $G$-absolutely negligible in $G$. 
Proof. Take any countable family $\left\{g_{i}: i \in I\right\}$ of elements of $G$ and denote $h_{i}=\phi\left(g_{i}\right)$ for all $i \in I$. Consider the set

$$
Y^{\prime}=\cup\left\{h_{i} Y: i \in I\right\} .
$$

Since $Y$ is $H$-absolutely negligible, there exists a countable family $\left\{h_{j}^{\prime}: j \in\right.$ $J\} \subset H$ such that

$$
\cap\left\{h_{j}^{\prime} Y^{\prime}: j \in J\right\}=\varnothing .
$$

Further, since $\phi$ is a surjection, we can find a countable family $\left\{g_{j}^{\prime}: j \in J\right\} \subset G$ such that $\phi\left(g_{j}^{\prime}\right)=h_{j}^{\prime}$ for all $j \in J$. Now, a straightforward verification shows that

$$
\bigcap_{j \in J} g_{j}^{\prime}\left(\bigcup_{i \in I} g_{i} X\right)=\varnothing .
$$

In view of Lemma 1 , we can conclude that $X$ is $G$-absolutely negligible in $G$. This ends the proof of Lemma 2.

Remark 1. There are several notions in the theory of invariant (quasiinvariant) measures which are closely connected with the concept of a $G$-absolutely negligible set. Among them let us especially mention the notion of a $G$-negligible set and the notion of a $G$-absolutely nonmeasurable set (for the precise definitions and more details, see [5]). We would like to underline that direct analogs of Lemma 2 are valid for those sets. Namely, let $G$ and $H$ be again two groups, let $\phi: G \rightarrow H$ be a surjective homomorphism and let $Y$ be a subset of $H$. Then we have:

(a) if $Y$ is $H$-negligible, then $\phi^{-1}(Y)$ is $G$-negligible;

(b) if $Y$ is $H$-absolutely nonmeasurable, then $\phi^{-1}(Y)$ is $G$-absolutely nonmeasurable.

These facts are useful in various constructions of $G$-negligible and $G$-absolutely nonmeasurable sets (cf. [6] and Chapter 14 in [7]).

Lemma 3. Let $(G, \cdot)$ be a group and let $H$ be a normal subgroup of $G$ such that $\operatorname{card}(G / H) \leq \omega$. Fix a selector $\left\{g_{k}: k \in K\right\}$ of the countable disjoint family of sets $G / H$. Suppose that $Y \subset H$ is an $H$-absolutely negligible subset of $H$. Then the set

$$
X=\cup\left\{g_{k} Y: k \in K\right\}
$$

turns out to be G-absolutely negligible in $G$.

Proof. We may assume without loss of generality that if $g_{k} \in H$, then $g_{k}$ coincides with the neutral element of $G$.

Consider an arbitrary countable family $\left\{g_{i}^{\prime}: i \in I\right\} \subset G$. Obviously, for each $i \in I$, we can write $g_{i}^{\prime}=g_{k(i)} \cdot h_{i}^{\prime}$ where $h_{i}^{\prime} \in H$.

Further, since $H$ is a normal subgroup of $G$, we have the inclusion

$$
\cup\left\{g_{i}^{\prime} X: i \in I\right\} \subset \cup\left\{g_{k} Z: k \in K\right\}
$$

where $Z=\cup\left\{h_{i}^{\prime \prime} Y: i \in I\right\}$ for some countable family $\left\{h_{i}^{\prime \prime}: i \in I\right\}$ of elements of $H$. Keeping in mind that $Y$ is $H$-absolutely negligible, we infer that there 
exists a countable subgroup $F$ of $H$ for which

$$
\cap\{f Z: f \in F\}=\varnothing .
$$

Consider the family

$$
U=\left\{g_{k} \cdot f: k \in K, f \in F\right\}
$$

of elements of $G$. This family is countable and we assert that

$$
\bigcap_{u \in U} u\left(\bigcup_{k \in K} g_{k} Z\right)=\varnothing
$$

Indeed, suppose otherwise, i.e. suppose that there exists an element

$$
t \in \bigcap_{u \in U} u\left(\bigcup_{k \in K} g_{k} Z\right) .
$$

Clearly, we can represent $t$ in the form $t=g_{k_{0}} \cdot h_{0}$ for some $k_{0} \in K$ and $h_{0} \in H$. Consequently, we have

$$
t=g_{k_{0}} \cdot h_{0} \in \bigcap_{f \in F} g_{k_{0}} f\left(\bigcup_{k \in K} g_{k} Z\right)
$$

from which it follows

$$
h_{0} \in \bigcap_{f \in F} f\left(\bigcup_{k \in K} g_{k} Z\right)
$$

or, equivalently,

$$
F \cdot h_{0} \subset \cup\left\{g_{k} Z: k \in K\right\} .
$$

Notice now that if $g_{k}$ does not belong to $H$, then $g_{k} Z \cap H=\varnothing$ and, therefore, $F \cdot h_{0} \cap g_{k} Z=\varnothing$. This circumstance implies at once the inclusion $F \cdot h_{0} \subset Z$ and hence the relation

$$
h_{0} \in \cap\{f Z: f \in F\}
$$

which contradicts the above-mentioned equality $\cap\{f Z: f \in F\}=\varnothing$. The obtained contradiction shows that

$$
\bigcap_{u \in U} u\left(\bigcup_{k \in K} g_{k} Z\right)=\varnothing
$$

and, consequently,

$$
\bigcap_{u \in U} u\left(\bigcup_{i \in I} g_{i}^{\prime} X\right)=\varnothing .
$$

The latter equality establishes the $G$-absolute negligibility of $X$. Lemma 3 has thus been proved.

Remark 2. For a commutative group $(G, \cdot)$ and for any its subgroup $H$ satisfying the relation $\operatorname{card}(G / H) \leq \omega$, a direct analog of Lemma 3 was formulated in Chapter 5 of the monograph [5] (without a proof).

Lemma 4. Let $(G, \cdot)$ be an arbitrary uncountable commutative group. There exists a countable covering of $G$ consisting of $G$-absolutely negligible sets. 

[5].

The proof of this lemma can be found in Chapter 5 of the same monograph

Now, we are able to formulate the following statement.

Theorem 1. Let $(G, \cdot)$ be an uncountable solvable group. There exists a countable covering of $G$ consisting of $G$-absolutely negligible sets.

Proof. Since our group $G$ is solvable, we have some composition series for this group:

$$
\{e\}=G_{0} \subset G_{1} \subset G_{2} \subset \ldots \subset G_{n}=G,
$$

where $e$ denotes the neutral element of $G$, each $G_{m}$ is a normal subgroup of $G_{m+1}$ and all factor groups $G_{m+1} / G_{m}$ are commutative.

We use the method of induction on $n$. For $n=1$, our group $G$ turns out to be commutative and we may directly apply Lemma 4 .

Suppose that the assertion of the theorem has already been proved for $n$ and let us establish its validity for $n+1$. Consider the two last groups $G_{n+1}=G$ and $G_{n}$ in the composition series for our $G$ and denote by

$$
\phi: G_{n+1} \rightarrow G_{n+1} / G_{n}
$$

the canonical surjective homomorphism. Only two cases are possible.

1. $\operatorname{card}\left(G_{n+1} / G_{n}\right)>\omega$. In this case, we apply Lemma 4 and cover the factor group $G_{n+1} / G_{n}$ by a countable family $\left\{Y_{p}: p \in P\right\}$ of $\left(G_{n+1} / G_{n}\right)$-absolutely negligible sets. According to Lemma 2, the family $\left\{\phi^{-1}\left(Y_{p}\right): p \in P\right\}$ is a countable covering of $G$ consisting of $G$-absolutely negligible sets.

2. $\operatorname{card}\left(G_{n+1} / G_{n}\right) \leq \omega$. In this case, we obviously have $\operatorname{card}\left(G_{n}\right)>\omega$. By virtue of the inductive assumption, there exists a countable family $\left\{Y_{p}: p \in P\right\}$ of $G_{n}$-absolutely negligible sets, such that

$$
G_{n}=\cup\left\{Y_{p}: p \in P\right\} .
$$

Denote by $\left\{g_{k}: k \in K\right\}$ a selector of $G_{n+1} / G_{n}$. In our situation $\operatorname{card}(K) \leq \omega$ and, in view of Lemma 3, all sets

$$
X_{p}=\cup\left\{g_{k} Y_{p}: k \in K\right\}(p \in P)
$$

are $G$-absolutely negligible. Finally, it is evident that $G=\cup\left\{X_{p}: p \in P\right\}$.

In both cases we get the required result and the theorem has thus been proved.

Remark 3. An analogous argument (the induction on the length of a composition series) works for establishing the existence of a $G$-absolutely nonmeasurable set in an arbitrary uncountable solvable group $(G, \cdot)$. The proof of this fact was first obtained in the paper [6] (see also Chapter 11 of the monograph [7]).

From Theorem 1 we readily deduce the following statement concerning strong extensions of invariant (quasi-invariant) measures on uncountable solvable groups.

Theorem 2. Let $(G, \cdot)$ be an uncountable solvable group and let $\mu$ be a nonzero $\sigma$-finite $G$-invariant (respectively, $G$-quasi-invariant) measure on $G$. 
Then there exists a $G$-invariant (respectively, G-quasi-invariant) measure on $G$ strictly extending $\mu$.

Remark 4. At the present time, we have some other methods of extending nonzero $\sigma$-finite $G$-invariant ( $G$-quasi-invariant) measures given on an uncountable group $(G, \cdot)$. One of these methods is essentially based on the concept of an almost $G$-invariant subset of $G$ (see, e.g., [4] and [5]). Namely, by using certain properties of almost $G$-invariant sets in $G$, it can be proved that if card $(G)$ is not a real-valued measurable cardinal, then for every nonzero $\sigma$-finite $G$-invariant (respectively, $G$-quasi-invariant) measure $\mu$ on $G$ there exists a $G$-invariant (respectively, $G$-quasi-invariant) measure $\mu^{\prime}$ on $G$ strictly extending $\mu$ (cf. [8], [9] and $[4])$.

\section{REFERENCES}

1. A. B. Kharazishvili, Certain types of invariant measures. (Russian) Dokl. Akad. Nauk SSSR 222(1975), No. 3, 538-540.

2. P. ERdös and R. D. Mauldin, The nonexistence of certain invariant measures. Proc. Amer. Math. Soc. 59(1976), No. 2, 321-322.

3. É. ŠPIL'RAĬN, On problems of the theory of measure. (Russian) Uspekhi Mat. Nauk (N.S.) 1(1946), No. 2(12), 179-188.

4. A. B. Kharazishvili, Invariant extensions of the Lebesgue measure. (Russian) Tbilis. Gos. Univ., Tbilisi, 1983.

5. A. B. Kharazishvili, Transformation groups and invariant measures. Set-theoretical aspects. World Scientific Publishing Co., Inc., River Edge, NJ, 1998.

6. A. B. Kharazishvili, Absolutely nonmeasurable sets in abelian groups. (Russian) Soobshch. Akad. Nauk Gruzin. SSR 97(1980), No. 3, 537-540.

7. A. B. Kharazishvili, Nonmeasurable sets and functions. North-Holland Mathematics Studies, 195. Elsevier Science B.V., Amsterdam, 2004.

8. S. S. Pkhakadze, The theory of Lebesgue measure. (Russian) Trudy Tbiliss. Mat. Inst. Razmadze 25(1958), 3-271.

9. A. Hulanicki, Invariant extensions of the Lebesgue measure. Fund. Math. 51(1962/ 1963), 111-115.

(Received 25.02.2004)

Author's address:

I. Vekua Institute of Applied Mathematics

I. Javakhishvili Tbilisi State University

2, University St., Tbilisi 0143

Georgia

E-mail: kharaz2@yahoo.com 